\title{
Threshold for Thermal Ionization of an Aluminum Surface by Pulsed Megagauss Magnetic Field
}

\author{
T. J. Awe, ${ }^{*}$ B. S. Bauer, S. Fuelling, and R. E. Siemon \\ University of Nevada, Reno, 5625 Fox Avenue, Reno, Nevada 89506, USA
}

(Received 26 June 2009; published 20 January 2010)

\begin{abstract}
The first measurement of the threshold for thermal ionization of the surface of thick metal by pulsed magnetic field $(B)$ is reported. Thick aluminum - with depth greater than the magnetic skin layer-was pulsed with $\partial B / \partial t$ from $30-80 \mathrm{MG} / \mu \mathrm{s}$. Novel loads avoided nonthermal plasma (from electron avalanche, or energetic particles or photons from arcs). Thermal plasma forms from 6061-alloy aluminum when the surface magnetic field reaches $2.2 \mathrm{MG}$, in qualitative agreement with numerical simulation results by Garanin et al. [J. Appl. Mech. Tech. Phys. 46, 153 (2005)].
\end{abstract}

DOI: 10.1103/PhysRevLett.104.035001

An important question for both basic science and applications is what state of matter is created from a thick metal surface Ohmically heated by an intense current pulse. The question of the conductivity of a metal surface under conditions of pulsed megagauss (MG) magnetic field has been posed since at least 1959 [1], when fields above 10 MG were produced by magnetic flux compression. A challenge has been uncertainty as to the state of the metal: liquid, vapor, warm dense matter, plasma, or a mixture of these. While considerable data exist for volumetrically heated thin wires [2-5], data have not been available for surface-heated thick metal. Plasma formation from Ohmically heated thick metal surfaces is much less certain than for thin wires, even for an order-of-magnitude higher linear current density. This is due in part to the continued availability of fresh underlying cold metal, whose high conductivity reduces the surface electric field and Ohmic heating to values that may be marginal for plasma to form. Although numerical modeling can describe how plasma heats on a surface [6], computational results can vary widely; for a magnetic-field pulse that rises to several MG, calculated peak temperatures may vary by orders of magnitude, from 0.1 to $100 \mathrm{eV}$. It is challenging to accurately model the interplay of magnetic diffusion, hydrodynamics, radiative energy transfer, and evolving material properties (e.g., resistivity varies by more than 10 orders of magnitude). Numerical predictions are particularly sensitive to numerical algorithms, material properties models, and computational parameters. This impacts a wide variety of applications, including ultrahigh magnetic-field generators, magnetized target fusion [7], transmission lines $[8,9]$, and magnetically accelerated flyer plates [10]. In this Letter, we report the first experimental measurement of the thermal ionization threshold of the surface of thick metal in response to a pulsed multi-MG magnetic field.

Studying if, when, where, and how thermal plasma is created by pulsed MG magnetic field, in the surfaceheating regime, poses a significant experimental challenge. Ultrahigh magnetic fields have been generated by imploding a conducting shell (liner) around a magnetic flux, for example, using high explosives [11] or high current [12].
PACS numbers: 52.25.-b, 07.55.Db, 52.50.-b, 52.58.Lq

Liquid, vapor, warm dense matter, and/or plasma may form on the inner liner wall as the compressed field reaches MG level [13]. The experiment reported here made multi-MG field by directly pulsing a high current on a conducting rod. This configuration enhances diagnostic access and reduces shot cost, but introduces new challenges. First, current pulses of substantial magnitude and rise rate are required to ensure that the penetration depth of the magnetic field is much smaller than the rod radius, and that multi-MG field is developed on the rod surface. In consequence, nonthermal plasma can be created due to electric-field-driven electron avalanche or photoionization from arcing electrical contacts. To avoid this, novel load hardware was developed and shown to mitigate nonthermal plasma production. The hardware design and performance is described below, after the current pulse requirements.

The 2-TW Zebra generator [14] is a suitable driver for experiments which examine the changes of state that occur in the interaction of pulsed multi-MG magnetic field with a thick conducting surface. For much of the data reported here the maximum current averaged $990 \mathrm{kA}$ with standard deviation $\sigma=30 \mathrm{kA}$, independent of the initial rod diameter. The Zebra current, $I(t)$, rose exponentially to $100 \mathrm{kA}$ (with rise time $\tau=13 \mathrm{~ns}$ ), and then linearly from 100 to $900 \mathrm{kA}$ for $70 \mathrm{~ns}$, with $d I / d t=1.1 \times$ $10^{13} \mathrm{~A} / \mathrm{s}$, to a maximum current of $1.0 \mathrm{MA}$. The skin depth starts at $0.017 \mathrm{~mm}$ in cold $\mathrm{Al}$, and grows to nearly $0.1 \mathrm{~mm}$ in hot, resistive Al. Zebra's parameters allow a range of $D_{0}$ around $1 \mathrm{~mm}$ to meet the surface-heating and high field requirements. Rod diameters have ranged from 2.00 to $0.50 \mathrm{~mm}$ with peak magnetic fields of 1.5 to $4 \mathrm{MG}$ (corresponding to $\partial B / \partial t$ of 30 to $80 \mathrm{MG} / \mu \mathrm{s}$ ) generated on the expanding surfaces. The observed consistency of the Zebra current waveform for modest changes in load-vacuum-chamber inductance (associated with changes in initial rod diameter) is supported by detailed modeling [15].

The experiment provides a meaningful comparison for one-dimensional (1D) MHD modeling. Novel load designs mitigate nonthermal plasma formation from contact arcing $[16,17]$ or electron avalanche. The low inductance of the 
load and large transmission line gap avoid nonthermal plasma formation from energetic particles or photons emitted from the transmission line or high-voltage insulator regions [18]. Because of the fast rise of the Zebra current pulse, rod surfaces exhibit (radially dependent) 1D behavior until plasma formation [19], with MHD instability observed only afterwards. Because of the importance of achieving thermal, uniform, and symmetric plasma formation, many different load geometries, contact configurations, and rod diameters were evaluated.

Load hardware was successfully designed to mitigate nonthermal plasma formation [20]. To avoid arcs, electrical connections were made at large diameter with buried, high-pressure metal-to-metal contacts. To avoid electric field enhancement, transitions to smaller radii were made slowly and smoothly. Three of the most commonly used load assemblies are depicted in Fig. 1. The simple straightrod load [1(a)] uses gravitationally seated anode and cathode plates, and has small-diameter slip-fit rod to anode-cathode electrical contacts. The "barbell" and "hourglass" loads [1(b) and 1(c)] connect to the power flow channel with "knife-edge" coupling hardware, which compress a large-diameter stainless steel ring through the aluminum oxide layer on the surface of the anode and cathode plates, decreasing the local contact resistance and current density. Barbell loads are machined from 6-mmdiameter cylinders to allow medium-diameter connections to the anode and cathode. Hourglass loads contain no contact points near the straight central section of the load, as the entire load is machined from a single piece of 3-inch-diameter $\mathrm{Al}$ stock.

Simple electrical contacts [Fig. 1(a)] generate arcs and nonuniform heating, with possible plasma formation by ultraviolet irradiation on rod surfaces far from the location of the arc. The response of contacts was characterized with imaged photomultipliers and an intensified, gated CCD camera. For the anode and cathode contacts of simple straight-rod loads [Fig. 1(a)], arcing begins at low current,

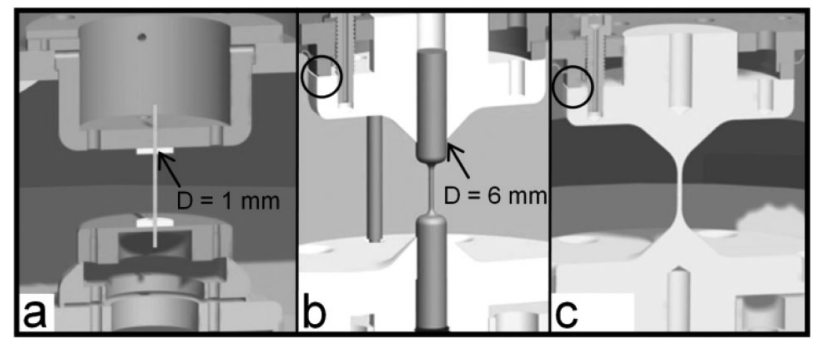

FIG. 1. Three load assemblies: (a) Simple straight-rod load with small diameter contacts and gravitationally established electrical connections; (b) "Barbell" load with large diameter contacts and compressed knife-edge connections; and (c) "Hourglass" load machined from a single piece of Al with compressed knife-edge connections. Straight central-rod sections of barbell and hourglass loads are $7 \mathrm{~mm}$ long. Circles locate knife-edge penetration points. and nonuniform plasma formation is observed on surfaces far from the current joints. In contrast, for barbell loads [Fig. 1(b)] no contact arcing is detected, and surface emission is much more spatially uniform. Hourglass loads [Fig. 1(c)] show surface emission uniformity similar to barbell loads.

Plasma is identified and characterized by a variety of independent measurements. Surface temperatures exceed $10 \mathrm{eV}$ for surface magnetic fields $\left(B_{s}\right)$ above $3 \mathrm{MG}$, as indicated by visible light radiometry. Multiply ionized Al is observed with time-resolved extreme ultraviolet (EUV) spectroscopy, and higher energy photons are detected with broadband EUV photodiodes. Plasma instabilities are recorded with gated imaging and laser shadowgraphy. The time-dependent value of $B_{s}$ is determined by time-resolved measurements of the discharge current and the surface radius. The plasma formation time, temperature profiles, and surface expansion characteristics vary with the surface magnetic-field rise rate $\left(\partial B_{s} / \partial t\right)$ and maximum $\left(B_{\max }\right)$, which increase when $D_{0}$ is decreased.

A lower bound on the surface temperature is obtained with visible light radiometry. The spectral radiation intensity escaping the rod is measured with a green-filtered linear diode array. The surface brightness temperature $\left(T_{B B}\right)$ is then calculated by using the Planckian blackbody formula. Time-resolved spectroscopy (a visible light imaging spectrograph coupled to a streak camera) supports the blackbody assumption: a line-free continuum is observed from 1.00-mm-diameter rods. Simulations suggest the surface plasma is optically thick to visible radiation [21]. An optically thin body radiates less than a perfect blackbody; therefore, $T_{B B}$ has low sensitivity to an optically thin corona.

Decreasing $D_{0}$ leads to earlier thermal plasma formation time (if plasma forms) and higher peak temperature. Peak $T_{B B}$ occur near the time of peak current, and reach 36,23 , $20,11,1.3$ and $0.7 \mathrm{eV}$ for $D_{0}$ of $0.50,0.80,1.00,1.25,1.59$, and $2.00 \mathrm{~mm}$, respectively. For $\mathrm{Al}$, multi-eV temperature clearly indicates plasma.

A second indicator of plasma was an EUV photon flux consistent with multi-eV temperatures, collected by photodiodes. Photodiodes filtered with $200 \mathrm{~nm}$ of $\mathrm{Al}$ or $100 \mathrm{~nm}$ of $\mathrm{Si}$ and $200 \mathrm{~nm}$ of $\mathrm{Zr}$, are sensitive to photon energies from 16 to $73 \mathrm{eV}$, or 60 to $100 \mathrm{eV}$, respectively. EUV diode signals (Fig. 2) start when $T_{B B} \sim 2.0 \mathrm{eV}$ (from optical diodes), and signal amplitudes are consistent with the observed $T_{B B}$ until the diodes saturate. The EUV flux was too low to be observed from 2.00-mm-diameter rods, consistent with a peak $T_{B B}$ of only $0.7 \mathrm{eV}$ (Fig. 2).

A third indicator of plasma was $\mathrm{Al}^{3+}$ and $\mathrm{Al}^{4+}$ line emission observed with time-resolved extreme ultraviolet (EUV) spectroscopy. EUV spectra (Fig. 3) in the band from 70 to $150 \mathrm{eV}$ were obtained with a McPherson 310/G grazing incidence spectrometer equipped with a multistrip MCP. PrismSPECT, a commercial collisional-radiative 


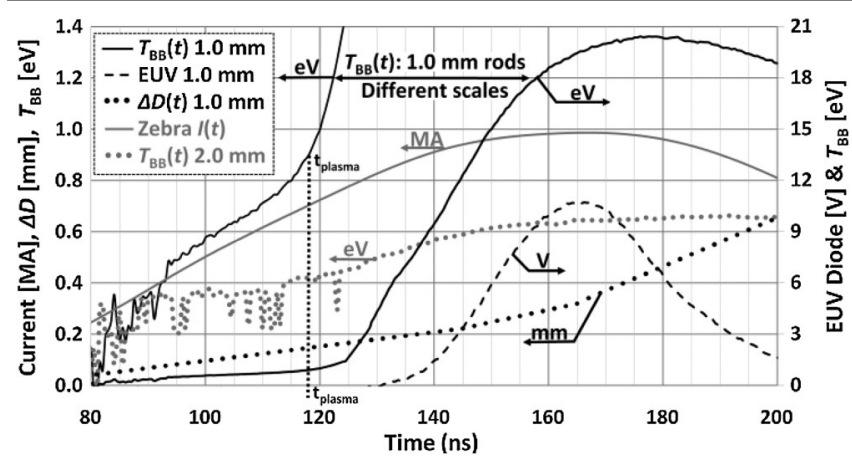

FIG. 2. $\quad T_{B B}(t)$ [eV], Al-filtered EUV diodes [V], and change in rod diameter $\Delta D(t)=D(t)-D_{0}$ [mm], for $1.00 \mathrm{~mm}$ rods, and $T_{B B}(t)[\mathrm{eV}]$ for $2.00 \mathrm{~mm}$ rods. Data are multishot averages. The average Zebra current is also plotted. Arrows (with units) designate the vertical scale for each curve. For $D_{0}=1.00 \mathrm{~mm}$, thermal plasma forms at $t_{\text {plasma }}=118 \mathrm{~ns}$, as indicated by the abrupt increase in $\partial T_{B B} / \partial t$. For $D_{0}=2.00 \mathrm{~mm}$, plasma does not appear to form.

spectral analysis code, was used to estimate the plasma temperature (Fig. 3). The average ion charge and line ratios depend strongly upon temperature, and taking the ratio of line intensities results in an estimated peak plasma temperature of $15 \pm 1 \mathrm{eV}$ for 1.00 -mm-diameter $\mathrm{Al}$ rods. Measurement uncertainty is much less than $1 \mathrm{eV}$, however, such precision is not realistic due to systematic uncertainty.

A fourth indicator of plasma was surface instabilities, whose development depends on the conductivity of the low density exploding material [22]. As indicated by timegated imaging and laser shadowgraphy (Fig. 4), instabilities grow on the surface of rods with $D_{0} \leq 1.25 \mathrm{~mm}$, and form earlier when $D_{0}$ is reduced.

A fifth possible indicator of plasma was a dramatic change at peak current of the surface radial expansion

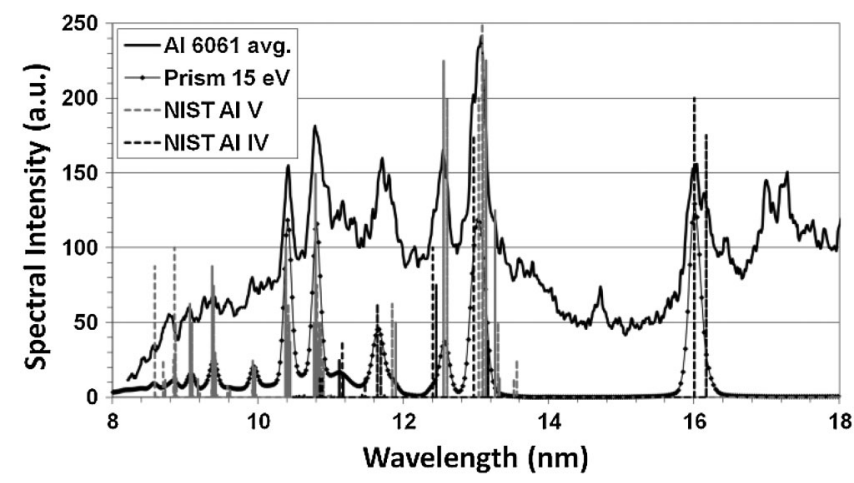

FIG. 3. Average EUV spectrum during 6 ns near peak current from 1.00-mm-diameter Al-6061 barbell loads in knife-edge hardware. The data (thick line) are presented along with the wavelength and magnitude of $\mathrm{Al}^{3+}$ and $\mathrm{Al}^{4+}$ lines obtained from the NIST Atomic Spectra Database (vertical dashed lines), and a PrismSPECT-calculated spectrum for $15 \mathrm{eV}$ temperature (thin line). PrismSPECT calculations assume a $1.0-\mu \mathrm{m}$-thick plasma layer with density $5 \times 10^{-3} \mathrm{~g} / \mathrm{cm}^{3}$. speed. Both 1.00 and 2.00-mm-diameter rods expand at a constant rate of $3 \mathrm{~km} / \mathrm{s}$ until near peak current. Then as the current drops, the surface velocity for 1.00 -mm-diameter rods exceeds $10 \mathrm{~km} / \mathrm{s}$, likely because of reduced magnetic pinching. In contrast, 2.00-mm-diameter rods remain at a constant $3 \mathrm{~km} / \mathrm{s}$ velocity as the current drops.

The onset of thermal plasma is evident in the optical diode signals, as an abrupt increase in $\partial T_{B B} / \partial t$ when $T_{B B}$ reaches 0.6 to $0.9 \mathrm{eV}$ for $D_{0} \leq 1.25 \mathrm{~mm}$ (shown for $1.00 \mathrm{~mm}$ rods, Fig. 2). The observed plasma formation temperature is consistent with calculations of the conductivity of warm, dense $\mathrm{Al}$ at below one-tenth solid density [23]. This supports determining the plasma formation time and magnetic-field threshold ( $\left.B_{\text {threshold }}\right)$ via the inflection in $T_{B B}(t)$.

Quite independent of $D_{0}$ (perhaps surprisingly), thermal plasma forms when $B_{s}$ reaches $B_{\text {threshold }}=2.2 \mathrm{MG}$. By decreasing $D_{0}$, the rate of energy deposition via Ohmic heating $P_{j}(r, t)=\eta(r, t)[j(r, t)]^{2} \approx \eta(r, t)\left\{I(t) /\left[\pi D_{0} \Delta(t)\right]\right\}^{2}$ increases [where $\Delta(t)$ is the increasing thickness of the current-carrying skin layer]. Melting, vaporization, and plasma formation occur earlier, at lower current. For plasma to form, Ohmic heating must exceed energy losses associated with expansion, thermal conduction, and radiation. For each $D_{0}$, the material takes a different trajectory through $\rho-T$ space, with many orders of magnitude change in resistivity and heat capacity within the skin layer, yet the formation of plasma remains highly correlated with the value of $B_{s}$. The surface magnetic field is calculated using the radius observed in gated imaging and laser backlighting, and the load current deduced from differential magnetic $(B$-dot $)$ probe measurements (Fig. 2). Rods with $D_{0}$ of $0.50,0.64,0.80,1.00$, and $1.25 \mathrm{~mm}$ form plasma when the $B_{s}$ reaches $2.0,2.1,2.2$, 2.3, and 2.2 MG, respectively. Plasma formation depends strongly upon $B_{s}$, but weakly upon magnetic-field rise rate $\left(\partial B_{s} / \partial t\right.$ decreasing from 80 to $\left.30 \mathrm{MG} / \mu \mathrm{s}\right)$ or upon nonplanar effects associated with small radius.

In conclusion, for $\partial B_{s} / \partial t$ of $30-80 \mathrm{MG} / \mu \mathrm{s}$, thermal plasma forms from an Al 6061-alloy surface when $B_{s}$

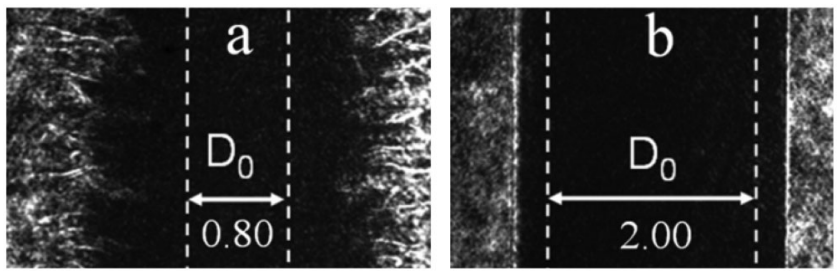

FIG. 4. Laser shadowgrams (resolution $150 \mathrm{ps}$ and $20 \mu \mathrm{m}$ ) for (a) $0.80-\mathrm{mm}$ and (b) 2.00 -mm-diameter rods, both at an instant near peak current $(1 \mathrm{MA})$. Dashed lines indicate $D_{0}$. The $0.80 \mathrm{~mm}$ rod forms surface plasma at low current that is highly unstable. In contrast, the $2.00 \mathrm{~mm}$ rod forms no surface plasma, and within the resolution limits of the instrument, shows no instability formation even after significant radial expansion. 
reaches 2.2 MG. The existence of a plasma formation threshold at the few MG level is in qualitative agreement with theoretical and numerical results [6]. The experiment offers the first detailed study of the threshold for plasma formation by pulsed magnetic fields on a thick $\mathrm{Al}$ surface. Nonthermal plasma production has been effectively mitigated with specialized load hardware. For $B_{s}$ below $2 \mathrm{MG}$, sub-eV temperatures persist, and no evidence of plasma is observed. Thermal plasma forms when $T_{B B}$ reaches approximately $0.7 \mathrm{eV}$ during the linear current rise, at which point rapid surface heating is observed. For rods with $D_{0}=$ $1.00 \mathrm{~mm}$, at peak current $B_{s}$ is $3.0 \mathrm{MG}, T_{B B}$ is $20 \mathrm{eV}$, and $\mathrm{Al}^{3+}$ and $\mathrm{Al}^{4+}$ ions are observed in approximately equal abundance. For a $\partial B_{s} / \partial t$ of $30-40 \mathrm{MG} / \mu \mathrm{s}, \mathrm{Al}$ surfaces expand at $3 \mathrm{~km} / \mathrm{s}$ during the current rise. The detailed measurements of phase, temperature, velocity, and ionization state of a thick metal surface as a function of intense pulsed magnetic field are informing radiation MHD modeling and will facilitate the design and engineering of practical devices.

The authors thank V. Makhin, I. Lindemuth, M. Angelova, W. Atchison, W. Cline, J. Degnan, M. Desjarlais, A. Esaulov, R. Faehl, M. Frese, S. Frese, S. Garanin, T. Goodrich, T. Haill, V. Ivanov, A. Kaul, B. Le Galloudec, D. Meredith, I. Paraschiv, R. Presura, R. Reinovsky, C. Rousculp, E. Ruden, and P. Turchi. Research supported by DOE grants DE-FG02-04ER54752, DEFG02-06ER54892, and DE-FC52-06NA27616.

*awetom@gmail.com

[1] C. M. Fowler, W. B. Garn, and R. S. Caird, J. Appl. Phys. 31, 588 (1960).
[2] Exploding Wires, edited by W. G. Chace and H. K. Moore (Plenum, New York, 1968), Vol. 4.

[3] S. V. Lebedev and A. I. Savvatimskii, Sov. Phys. Usp. 27, 749 (1984).

[4] M. G. Haines et al., Phys. Plasmas 7, 1672 (2000).

[5] D. A. Hammer and D. B. Sinars, Laser Part. Beams 19, 377 (2001).

[6] S. F. Garanin et al., J. Appl. Mech. Tech. Phys. 46, 153 (2005).

[7] I. R. Lindemuth and R.E. Siemon, Am. J. Phys. 77, 407 (2009).

[8] W. A. Stygar et al., Phys. Rev. E 72, 026404 (2005).

[9] S. A. Slutz, C. L. Olson, and P. Peterson, Phys. Plasmas 10, 429 (2003)

[10] R. W. Lemke et al., J. Appl. Phys. 98, 073530 (2005).

[11] C. M. Fowler, Science 180, 261 (1973).

[12] N. Miura et al., Int. J. Mod. Phys. B 16, 3379 (2002).

[13] P. J. Turchi, IEEE Trans. Plasma Sci. 28, 1414 (2000).

[14] B.S. Bauer et al., Proceedings of the 4th International Conference on Dense Z-Pinches, 1997 (AIP, Woodbury, NY, 1999), Vol. 409, p. 153.

[15] A. S. Chuvatin et al., Proceedings of the 7th International Conference on Dense Z-Pinches (AIP, Melville, NY, 2009), Vol. 1088, p. 253.

[16] P. U. Duselis, J. A. Vaughan, and B. R. Kusse, Phys. Plasmas 11, 4025 (2004).

[17] T. W. L. Sanford et al., Phys. Plasmas 12, 122701 (2005).

[18] M.E. Cuneo, IEEE Trans. Dielectr. Electr. Insul. 6, 469 (1999).

[19] R. E. Siemon et al., J. Fusion Energy 27, 235 (2008).

[20] S. Fuelling et al., IEEE Trans. Plasma Sci. 36, 62 (2008).

[21] S. Mazevet et al., Phys. Rev. E 71, 016409 (2005).

[22] M. A. Angelova et al., IEEE Trans. Plasma Sci. 36, 37 (2008).

[23] M. P. Desjarlais, J. D. Kress, and L. A. Collins, Phys. Rev. E 66, 025401(R) (2002). 